\title{
Metal-insulator transition in EuO
}

\author{
P. Sinjukow ${ }^{1, *}$ and W. Nolting ${ }^{1}$ \\ ${ }^{1}$ Lehrstuhl Festkörpertheorie, Institut für Physik, Humboldt-Universität zu Berlin, Newtonstr. 15, 12489 Berlin
}

(Dated: August 13, 2018)

\begin{abstract}
It is shown that the spectacular metal-insulator transition in Eu-rich EuO can be simulated within an extended Kondo lattice model. The different orders of magnitude of the jump in resistivity in dependence on the concentration of oxygen vacancies as well as the low-temperature resistance minimum in high-resistivity samples are reproduced quantitatively. The huge colossal magnetoresistance (CMR) is calculated and discussed.
\end{abstract}

\section{INTRODUCTION}

Stoichiometric (pure) EuO is a ferromagnetic semiconductor. In the sixties and seventies it became famous for the redshift of its optical absorption edge below the Curie temperature $T_{C}(69 \mathrm{~K})^{1}$. Eu-rich EuO, whose Eu richness is realized by oxygen vacancies, behaves like a metal at low temperatures and shows a tremendous metalinsulator transition near the Curie temperature 2.3.4. A jump in resistivity of 13 orders of magnitude was mea-

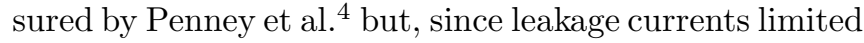
the measurement, it was probably even greater. It is a remarkable feature that the size of the jump in resistivity varies greatly amongst different experimental samples ${ }^{3}$. Another intriguing feature is a low-temperature resistance minimum in high-resistivity samples. Furthermore, Eu-rich EuO has a huge colossal magnetoresistance $(\mathrm{CMR}) \underline{\underline{5}}$.

Recently interest in $\mathrm{EuO}$ was renewed by measurements of the resistance and of the spin-split conduction band of a EuO film by Steeneken et al $\stackrel{6}{.}$. The authors emphasize the extraordinary properties of Eu-rich EuO and their importance for basic research in the field of spintronics due to the almost perfect spin polarization of the conduction electrons at low temperatures.

The first physical picture (He model) for the metalinsulator transition in Eu-rich EuO was developed by Oliver et $a l^{2}$. They postulated a temperatureindependent electronic trap (impurity) level created by the oxygen vacancies. It is below the conduction band for high temperatures, where Eu-rich $\mathrm{EuO}$ is an insulator. On decreasing the temperature below $T_{C}$, the spin-up conduction band is shifted towards lower energies (redshift) and therefore crosses the impurity level at some point. The electrons of the trap level are emptied into the conduction band giving rise to EuO becoming a metal. Later the model was refined by introducing a Coulomb repulsion between the two electrons at an oxygen vacancy and ascribing a temperature-dependent shift to the trap levels ${ }^{3}$. However, the shift of the impurity levels is unphysical as will be explained below. There is a second physical picture (the bound magnetic polaron (BMP)),

*Electronic address: sinjukow@physik.hu-berlin.de which was introduced by Torrance et al $\stackrel{7}{?}$. They assumed an exchange interaction of the surplus electrons of each vacancy site with the Eu spins. Theoretical attempts to quantify the metal-insulator transition of Eu-rich EuO were made by Laks et al $\stackrel{8}{*}$, Mauger ${ }^{9}$ and Steeneken ${ }^{10}$. The conductivity in all three cases was obtained by the simple Drude formula $\sigma=n_{c} e \mu\left(n_{c^{-}}\right.$conduction-electron density, $e$-elementary charge, $\mu$-mobility).

In this paper we formulate an extended Kondo-lattice Hamiltonian as a microscopic model for the metalinsulator transition in Eu-rich EuO. It contains qualitatively the idea of the He model through the assumption of impurity trap levels, and it contains the physics of the BMP since an exchange between the impurity-electron spins and the Eu spins is included. However, our formulation goes beyond the former two approaches because of the detailed modelling of the impurity orbital energies and their hybridization with the conduction band. The disorder of the oxygen vacancies is treated within an extension of the coherent-potential approximation (CPA). Our calculations should be more accurate than the ones in Refs. 8.910 since we are using a more complex conductivity formula ${ }^{11,12}$, which is derived directly from the fundamental current-current correlation Kubo formula.

\section{THEORY}

In order to model the situation in Eu-rich EuO, first one has to consider a conduction band $\epsilon_{\vec{k}}$. The spins of the conduction electrons $\vec{\sigma}_{i}$ are coupled to the Eu spins $\vec{S}_{i}$ via a coupling constant $J$. These terms represent the Kondo lattice model. Then, there are impurity levels $\epsilon_{p}$, a Coulomb repulsion $U$ in case of two electrons at an oxygen vacancy, and an exchange between the impurityelectron spins $\vec{\sigma}_{j}^{p}$ and the Eu spins with coupling constant $J_{p}$. Furthermore, there should be a hybridization $V$ between impurity electrons $\left(p_{j \sigma}^{\dagger}\right)$ and conduction electrons $\left(c_{j \sigma}^{\dagger}\right)$. The superexchange between the Eu spins is not modeled explicitly. Instead we are considering $\left\langle S_{z}\right\rangle$ as a parameter, whose temperature dependence is given by a Brillouin function, which is fulfilled to very high accuracy in Eu-rich $\mathrm{EuO}^{13}$. The Hamiltonian which captures all 
the features discussed is the following:

$$
\begin{aligned}
H= & \sum_{\vec{k} \sigma} \epsilon_{\vec{k}} n_{\vec{k} \sigma}-J \sum_{i} \vec{S}_{i} \cdot \vec{\sigma}_{i} \\
& +\sum_{j \sigma} \epsilon_{p} n_{j \sigma}^{p}+U \sum_{j} n_{j \uparrow}^{p} n_{j \downarrow}^{p}-J_{p} \sum_{j} \vec{S}_{j} \cdot \vec{\sigma}_{j}^{p} \\
& +V \sum_{j \sigma}\left(p_{j \sigma}^{\dagger} c_{j \sigma}+c_{j \sigma}^{\dagger} p_{j \sigma}\right)
\end{aligned}
$$

The sums over $i$ and $j$ mean a sum over all lattice sites and a sum over the randomly distributed oxygen-vacancy (impurity) sites, respectively. As the actual value of $J_{p}$ is not known, $J_{p}=J$ is assumed for simplicity.

The current operator is given by

$$
j=\lim _{\vec{q} \rightarrow 0} \frac{e}{|\vec{q}|}[H, \rho(\vec{q})]
$$

with the density operator ${ }^{14}$

$$
\rho(\vec{q})=\sum_{\vec{k} \sigma} c_{\vec{k}-\vec{q} \sigma}^{\dagger} c_{\vec{k} \sigma}+\sum_{j \sigma} e^{-i \vec{q} \vec{R}_{j}} p_{j \sigma}^{\dagger} p_{j \sigma}+O\left(q^{2}\right) .
$$

Eqs. (11)-(3) yield a surprisingly simple result for the current operator in a cubic system:

$$
j=-e \sum_{\vec{k} \sigma} \frac{\partial \epsilon_{\vec{k}}}{\partial k_{x}} c_{\vec{k} \sigma}^{\dagger} c_{\vec{k} \sigma} .
$$

Eq. (44) has to be put into the current-current correlation Kubo formula for the conductivity ${ }^{15}$. If the self-energy is local, which is exact in infinite dimensions, e.g. if applying dynamical-mean field theory (DMFT), there is a substantial simplification to the Kubo formula. All vertex corrections to the current-current correlation function vanish and one is left with an expression that only contains the one-particle spectral density ${ }^{11,12}$. We apply the local approximation to the self-energy and use the same conductivity formula for the three-dimensional case:

$$
\sigma=\frac{e^{2} \pi}{\hbar V} \sum_{\vec{k} \sigma} \int_{-\infty}^{\infty} d E\left(-f^{\prime}(E)\right) A_{\vec{k} \sigma}(E)^{2}\left(\frac{\partial \epsilon_{\vec{k}}}{\partial k_{x}}\right)^{2}
$$

where $V$ is the volume, $f^{\prime}(E)$ is the derivative of the Fermi function and $A_{\vec{k} \sigma}(E)$ is the spectral density of the conduction electrons. Eq. (5) can be transformed ${ }^{16}$ into

$$
\begin{aligned}
& \sigma=\frac{e^{2} \pi}{6 \hbar a} \sum_{\sigma} \int_{-\infty}^{\infty} d E\left(-f^{\prime}(E)\right) \int_{-\infty}^{\infty} d x \phi(x, E) \hat{v}(x) \\
& \phi(x, E)=A_{\vec{k} \sigma}(E)_{\epsilon_{\vec{k}} \rightarrow x}^{2} \\
& \hat{v}(x)=-\int_{-\infty}^{x} d E^{\prime} E^{\prime} \rho_{0}\left(E^{\prime}\right) .
\end{aligned}
$$

$a$ is the lattice constant $(=5.1 \AA$ in $\mathrm{EuO})$, and $\rho_{0}(E)$ is the free conduction band density of states.
To get the spectral density $A_{\vec{k} \sigma}(E)$, we introduce appropriate selfenergies:

$$
\begin{aligned}
& \left\langle\left\langle\left[c_{\vec{k} \sigma},-J \sum_{i} \vec{S}_{i} \cdot \vec{\sigma}_{i}\right]_{-} ; c_{\vec{k} \sigma}^{\dagger}\right\rangle\right\rangle=\Sigma_{\vec{k} \sigma}(E)\left\langle\left\langle c_{\vec{k} \sigma} ; c_{\vec{k} \sigma}^{\dagger}\right\rangle\right\rangle \\
& \left\langle\left\langle\left[p_{j^{\prime} \sigma}, U \sum_{j} n_{j \uparrow}^{p} n_{j \downarrow}^{p}-J \sum_{j} \vec{S}_{j} \cdot \vec{\sigma}_{j}^{p}\right]_{-} ; p_{j^{\prime} \sigma}^{\dagger}\right\rangle\right\rangle \\
& =\Sigma_{\sigma}^{p}(E)\left\langle\left\langle p_{j^{\prime} \sigma} ; p_{j^{\prime} \sigma}^{\dagger}\right\rangle\right\rangle .
\end{aligned}
$$

$\langle\langle\ldots ; \ldots\rangle\rangle$ stands for the retarded Green's function. Eqs. (77) and (8) are so far exact relations.

The main approximation of our approach consists in making independent ansatzes for the conduction-electron and impurity selfenergies. This is justified if the effect of the hybridization, which to the lowest order is proportional to $V^{2}$, is small. In our calculations it turns out that the hybridization $V$ itself has to be small to give a reasonable order of magnitude of the metal-insulator transition. Therefore, it is reasonable to neglect in the selfenergies effects of the order of $V^{2}$.

The conduction-electron self-energy is taken from an interpolating ansatz ${ }^{17}$ for the conduction electron part of the Hamiltonian (11) $\left(\sum_{\vec{k} \sigma} \epsilon_{\vec{k}} n_{\vec{k} \sigma}-J \sum_{i} \vec{S}_{i} \cdot \vec{\sigma}_{i}\right)$ :

$\Sigma_{\sigma}(E)=-\frac{1}{2} z_{\sigma} J\left\langle S^{z}\right\rangle+\frac{1}{4} J^{2} \frac{a_{\sigma} G_{0}\left(E+\mu-\frac{1}{2} z_{\sigma} J\left\langle S^{z}\right\rangle\right)}{1-b_{\sigma} G_{0}\left(E+\mu-\frac{1}{2} z_{\sigma} J\left\langle S^{z}\right\rangle\right)}$

$a_{\sigma}=S(S+1)-z_{\sigma}\left\langle S^{z}\right\rangle\left(z_{\sigma}\left\langle S^{z}\right\rangle+1\right), \quad b_{\sigma}=\frac{1}{2} J$

where $z_{\sigma}=\delta_{\sigma \uparrow}-\delta_{\sigma \downarrow}$ and $G_{0}(E)$ is the free conduction electron Green's function. The ansatz (9) fulfills all known limiting cases for $n_{c} \rightarrow 0$ (ferromagnetic saturation, atomic limit, second-order perturbation theory in $J$ and high-energy-expansion up to the fourth moment). It is therefore especially appropriate in the present case of very small conduction-electron densities $\left(10^{-3}-10^{-4}\right.$ per unit cell).

For the impurity self-energy $\Sigma_{\sigma}^{p}(E)$ we take the selfenergy of the atomic limit of the correlated Kondolattice model 18 (impurity part of the Hamiltonian (10) $\left(\sum_{j \sigma} \epsilon_{p} n_{j \sigma}^{p}+U \sum_{j} n_{j \uparrow}^{p} n_{j \downarrow}^{p}-J_{p} \sum_{j} \vec{S}_{j} \cdot \vec{\sigma}_{j}^{p}\right)$. If $U$ is large enough in comparison with $J$, this leads to a 4-peak structure in the impurity quasiparticle density of states with the highest peak at $\epsilon_{4}=\epsilon_{p}+U+\frac{J}{2} S$. The peak positions are fixed, i.e. do not depend on the magnetization $\left\langle S_{z}\right\rangle$. Therefore, the above-mentioned picture by Oliver et $\mathrm{al}^{\underline{3}}$, , which asigns a temperature-dependent shift to the impurity levels, is wrong. However, the weights of the peaks depend on $\left\langle S_{z}\right\rangle$ as well as on the impurity occupation. For any parameters only 3 peaks have a finite weight. The peak at $\epsilon_{4}$ will act as the impurity level which donates electrons to the conduction band.

We have made ansatzes for the selfenergies $\Sigma_{\sigma}^{p}(E)$ and $\Sigma_{\sigma}(E)$ which are correct for all known limiting cases of $n_{c} \rightarrow 0$ and up to the first order in the hybridization $V$. 
To treat the randomness of the oxygen vacancies we use the coherent-potential approximation (CPA), which originally was invented to deal with a non-interacting alloy ${ }^{19}$. Here we are dealing with strongly interacting electrons in a two-component alloy. One component corresponds to the impurity sites $A$, the other to the nonimpurity sites $B$. First, because of our effective medium approach and the locality of the impurity self-energy, local effective one-particle energies for the impurity sites are retained. The second problem is how to treat the non-impurity sites in a consistent way. To solve this, for non-impurity sites we also introduce one-particle levels $\sum_{\sigma} \epsilon_{p, i} n_{i \sigma}^{p}$ and hybridization terms with the conduction band $V \sum_{\sigma}\left(p_{i \sigma}^{\dagger} c_{i \sigma}+c_{i \sigma}^{\dagger} p_{i \sigma}\right)$. The one-particle energies $\epsilon_{p, i}$ must diverge to infinity at non-impurity sites to assure that these levels are never really occupied. With these virtual levels and a non-random hybridization on the lattice the usual CPA equation can be formulated:

$0=\sum_{m=A, B} c_{m} \frac{\epsilon_{p, m}-\mu+\Sigma_{m \sigma}^{p}(E)-\Sigma_{\sigma}^{\mathrm{CPA}}(E)}{1-G_{\sigma}^{p}(E)\left(\epsilon_{p, m}-\mu+\Sigma_{m \sigma}^{p}(E)-\Sigma_{\sigma}^{\mathrm{CPA}}(E)\right)}$

$$
\epsilon_{p, m}=\left\{\begin{array}{ll}
\epsilon_{p} & m=A \\
\infty & m=B
\end{array} \quad, \quad \Sigma_{m \sigma}^{p}=\left\{\begin{array}{cc}
\Sigma_{\sigma}^{p} & m=A \\
0 & m=B
\end{array}\right.\right.
$$

with $c_{A}=d$ and $c_{B}=1-d$ the concentrations of impurity and non-impurity sites, respectively. $G_{\sigma}^{p}$ is the local impurity Green's function and $\Sigma_{\sigma}^{\mathrm{CPA}}$ the CPA self-energy. The CPA is known to be correct up to the first order in the impurity concentration $d^{20}$. The Green's function of the conduction electrons is given by

$$
G_{\vec{k} \sigma}(E)=\frac{1}{E-(\epsilon(\vec{k})-\mu)-\frac{V^{2}}{E-\Sigma_{\sigma}^{C P A}(E)}-\Sigma_{\sigma}(E)} .
$$

From Eq. 11]the spectral density $A_{\vec{k} \sigma}(E)$ can be obtained. Since $A_{\vec{k} \sigma}(E)^{2}$ enters the conductivity formula (5), our calculations of the resisitivity are correct for all limiting cases of $n_{c} \rightarrow 0$ and up to the second order in the hybridization $V$ and the impurity concentration $d$.

The parameters for the calculations are as follows. The width of the conduction band $W(\approx 10 \mathrm{eV})$ can be taken from the absorption spectrum of Ref. $[6$ or from band structure calculations ${ }^{21}$. We have assumed a semielliptical shape of the conduction band, which approximates well the actual situation at the lower band edge 21 . $J$ determines the shift of the conduction band $\left(\frac{J}{2} S\right)$, which is about $0.3 \mathrm{eV}$ (half band-splitting) at low temperatures ${ }^{6}$. For Eu spins of $S=\frac{7}{2}$ this implies a coupling constant $J=0.17 \mathrm{eV}$. The position of $\epsilon_{4}$ is in general not known. Only for the highest-resistivity curves an activation energy of $0.3 \mathrm{eV}$ was observed ${ }^{3.7}$. If $U$ is assumed to be fixed at $1 \mathrm{eV}$, the impurity energy $\epsilon_{p}$ remains the parameter to be adjusted. The hybridization $V$, which is also not known, should not be too large in order not to destroy the effect of the metal-insulator transition. We have chosen $V=0.01 \mathrm{eV}$. Of all parameters, $W$ and $J$ are taken from the experiment. The precise values of $U$ and $V$ are not important apart from the fact that the hybridization should be small. The decisive free parameter is the impurity level $\epsilon_{4}$ since it determines the position of the chemical potential. For the vacancy (impurity) concentration $d$ medium values are of the order of $0.1 \%$. 6 . Two electrons are assigned to each oxygen vacancy.

\section{RESULTS AND DISCUSSION}

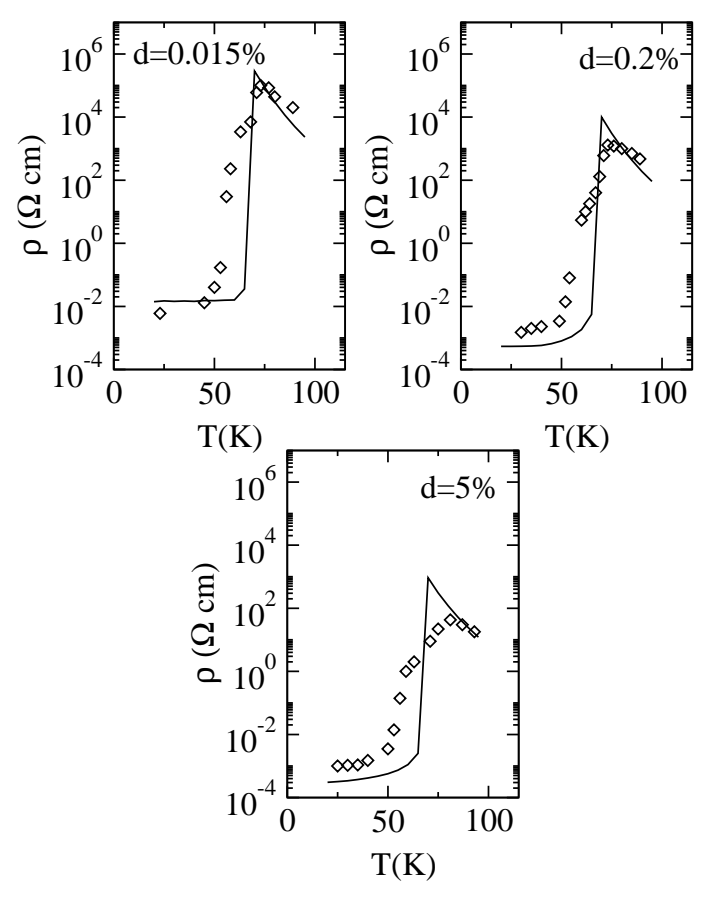

FIG. 1: Comparison of measured (diamonds, samples 66-6, 95-BA-3 and 49-4 from Ref. ${ }^{3}$ ) and calculated resistivity (solid lines) in dependence on temperature. The theoretical curves are calculated for three different impurity concentrations $d$. $\epsilon_{p}=-6.44 \mathrm{eV}\left(\epsilon_{4}=-5.14 \mathrm{eV}\right.$, lower band edge at $-5.30 \mathrm{eV}$ at $T=0)$. For other parameters see the text.

In Fig. 1 a comparison of calculated and measured resistivity curves is shown. The measured curves represent three examples of moderate resistivity from Ref. 3. The only varying parameter of the three theoretical curves is the vacancy concentration $d . \epsilon_{p}$ (and with it $\epsilon_{4}$ ) was fixed so as to yield the best overall agreement with the experimental curves. A variation of $\epsilon_{p}\left(\epsilon_{4}\right)$ has to be taken into account in order to explain the resistance minimum in high-resistivity samples (see below). Each curve in Fig. 1] shows a huge metal-insulator transition of several orders of magnitude near the magnetic transition temperature. The resistivity in the whole temperature range gets higher and the jump in resistivity at $T_{c}$ bigger, the fewer oxygen vacancies (electron donors) there 
are. Note the different orders of magnitude (7,6 and 5) of the jump in resistivity of the three experimental samples, which together with the absolute values of the resistivity are reproduced fairly well by the calculated curves. This demonstrates that the variation in the resistivity behaviour can be explained by different impurity concentrations. Incidentally, there is almost a $100 \%$ spin polarization of the conduction electrons at low temperatures as reported by Steeneken et al,$\underline{6}$, which is important for possible applications in spintronics.
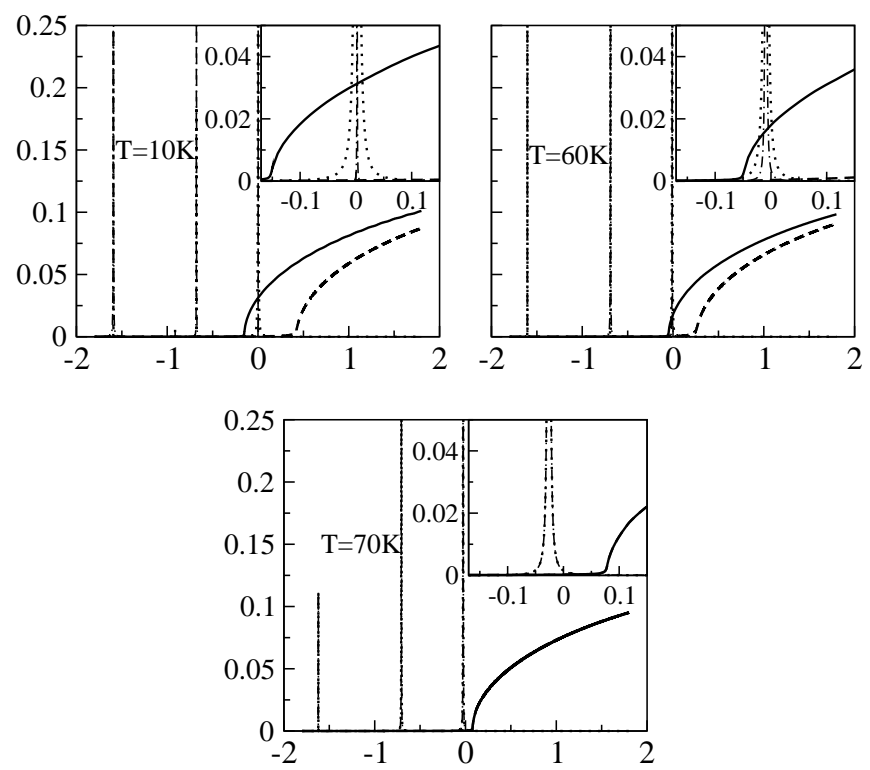

FIG. 2: Conduction-electron densities of states $\left(\rho_{\uparrow}(-), \rho_{\downarrow}(--)\right)$ and impurity densities of states $\left(\rho_{p \uparrow}(-\cdot-), \rho_{p \downarrow}(\cdots)\right)$ for $\epsilon_{p}=-6.44 \mathrm{eV}, d=0.05 \%$ and three different temperatures. For other parameters see the text. The insets show the densities of states near the Fermi level.

A qualitative explanation of the metal-insulator transition is possible in terms of quasiparticle densities of states along the lines of the He model (with the difference that there are three instead of one impurity level, so the number of electrons which can be emptied into the conduction band is not so big). As shown in Fig. 2 for low temperatures the uppermost impurity level $\epsilon_{4}$ lies within the spin-up part of the conduction band, which is down-shifted due to the exchange with the Eu spins. Therefore, a part of the impurity electrons is emptied into the conduction band. The system is a metal. On increasing the temperature the conduction band is shifted upwards. The number of conduction electrons decreases and the resistivity rises. Above the Curie temperature the conduction band is well separated from the uppermost impurity level, so only thermally excited electrons contribute to the conductivity, like in an n-doped semiconductor. The resistivity is high and decreases with increasing temperature. Hence, the system is an insulator.

For a quantitative analysis of the metal-insulator tran- sition we rely on the conductivity formula Eq. (6). The energy integrals in that formula yield a highly non-linear dependence of the conductivity on the number of conduction electrons $n_{c}$, which goes beyond the simple Drude formula $\sigma=n_{c} e \mu$ applied by other authors.

For high-resistivity samples there is a characteristic low-temperature minimum in the resistivity ${ }^{3}$. Oliver et

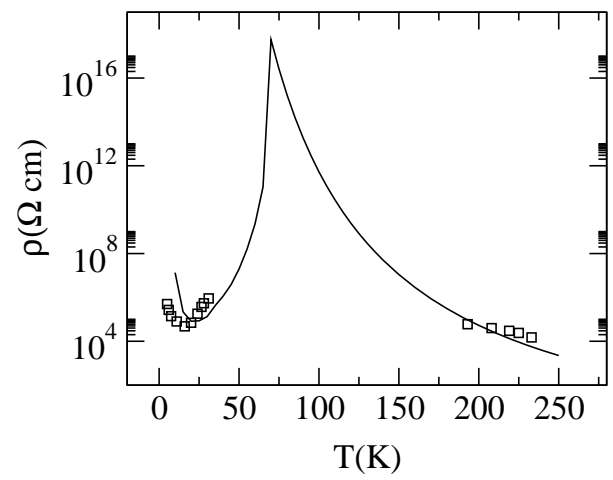

FIG. 3: Comparison of measured (squares, sample 34-2-30 from Ref ${ }^{3}$ ) and calculated resistivity (solid line) for a highresistivity sample. Impurity concentration $d=0.01 \%, \epsilon_{p}=$ $-6.61 \mathrm{eV}\left(\epsilon_{4}=-5.31 \mathrm{eV}\right.$, lower band edge at $-5.30 \mathrm{eV}$ at $T=$ $0)$. For other parameters see the text.

al ${ }^{3}$ explained it by activation from additional impurities like Gd or La. However, a more direct explanation is possible within our model without assuming additional impurities. The high values of the resistivity indicate that the impurity peak at $\epsilon_{4}$ must be rather distant from the conduction band at $T_{C}$. On lowering the temperature, the resistivity first decreases since the spin-up conduction band moves down towards the impurity level $\epsilon_{4}$. More electrons are thermally excited from the impurity level into the conduction band. If the position of $\epsilon_{4}$ is low enough never to cross the conduction band, then on lowering the temperature further, the resistivity increases again near the ferromagnetic saturation $\left\langle S_{z}\right\rangle \approx S$. When the conduction band stays almost fixed with respect to the impurity level, fewer electrons are excited into the conduction band with decreasing temperature. To test this explanation, we have calculated the temperaturedependent resistivity for $\epsilon_{p}=-6.61 \mathrm{eV}\left(\epsilon_{4}=-5.31 \mathrm{eV}\right.$, lower band edge at $-5.30 \mathrm{eV}$ at $T=0$ ) and impurity concentration $d=0.01 \%$. In Fig. B the calculated and a measured ${ }^{3}$ curve for a high-resistivity sample are shown. The theoretical curve has a minimum at $T=20 \mathrm{~K}$ which fits quite well to the experimental one. Moreover, there is a quantitative agreement between the high-temperature tails of the experimental and the calculated curves. For the temperature range in between no measured points are available but the run of the theoretical curve seems credible although it takes on very high values. (Penney et al.'s above-mentioned resistivity measurements ${ }^{\underline{4}}$ were limited to values of $10^{11} \Omega \mathrm{cm}$ but interpolating their data allows values of up to $10^{16} \Omega \mathrm{cm}$.)

The calculated dependence of the resistivity on a mag- 
netic field $B$ is shown in the left graph of Fig. 4 It looks qualitatively similar to the measured dependence in Fig. 3 of Ref. 5. A characteristic shift of the resistivity
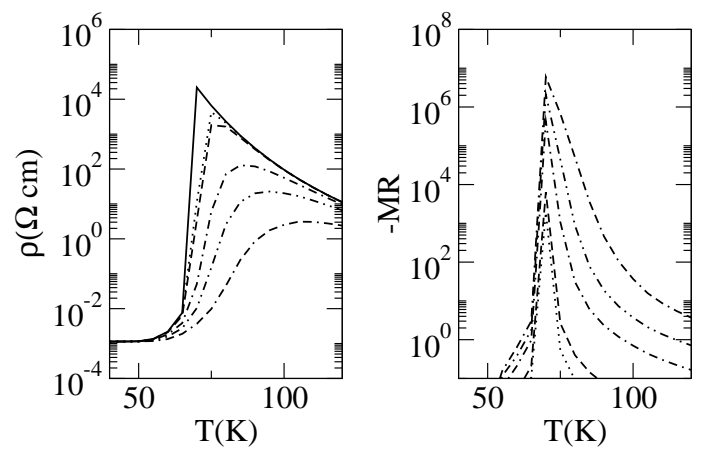

FIG. 4: Left: Calculated resistivity for different magnetic fields $B$; right: negative magnetoresistance. $B=0 \mathrm{~T}(-)$, $0.05 \mathrm{~T}(\cdots), 0.1 \mathrm{~T}(--), 0.5 \mathrm{~T}(-\cdot-\cdot), 1 \mathrm{~T}(-\cdot \cdot-\cdot), 2 \mathrm{~T}(\cdot--\cdot)$. $d=0.1 \%$; for other parameters see the text.

maximum from $T_{C}$ towards higher temperatures can be observed. This is due to the shift of the conduction band depending on the magnetization. Applying a magnetic field has a similar effect as lowering the temperature. At the same time the value of the maximum decreases significantly since the metal-insulator transition is smeared out because of the higher temperature of the maximum. Therefore there is a huge negative magnetoresistance MR $=\frac{\rho(B)-\rho(0)}{\rho(B)}$ as shown in the right graph of
Fig. [1 If one normalizes the magnetoresistance with $\rho(0)$ instead of $\rho(B)$, the magnetoresistance will be practically 1 in a wide temperature range. This can be compared with a value of only 0.8 for the colossal magnetoresistance (CMR) of the manganites like $\mathrm{La}_{1-x} \mathrm{Ca}_{x} \mathrm{MnO}_{3}{ }^{22}$.

\section{CONCLUSIONS}

In summary, a model has been presented which, in connection with a conductivity formula that is derived from the fundamental Kubo formula in the local-self-energy approximation, allows to accurately reproduce the spectacular metal-insulator transition and the huge CMR in $\mathrm{Eu}$-rich $\mathrm{EuO}$. The oxygen vacancy concentration is the decisive parameter for the big variations in resistivity behaviour. It may influence the position of the uppermost impurity level, which is important in order to explain the resistance minimum in high-resistivity samples. The precise dependence of the resistivity on both the impurity concentration and the position of the impurity level should be a subject of thorough future experimental investigation, especially if aiming at applications in the field of spintronics.

\section{Acknowledgments}

This work was supported by the Deutsche Forschungsgemeinschaft (Sfb 290).
${ }^{1}$ P. Wachter, Handbook on the Physics and Chemistry of Rare Earths (K. A. Gschneidner and L. Eyring, Elsevier, Amsterdam, 1979), vol. 2, p. 507.

2 M. R. Oliver, J. A. Kafalas, J. O. Dimmock, and T. B. Reed, Phys. Rev. Lett. 24, 1064 (1970).

3 M. R. Oliver, J. O. Dimmock, A. L. McWhorter, and T. B. Reed, Phys. Rev. B 5, 1078 (1972).

4 T. Penney, M. W. Shafer, and J. B. Torrance, Phys. Rev. B 5, 3669 (1972).

5 Y. Shapira, S. Foner, and T. B. Reed, Phys. Rev. B 8, 2299 (1973).

${ }^{6}$ P. G. Steeneken, L. H. Tjeng, I. Elfimov, G. A. Sawatzky, G. Ghiringhelli, N. B. Brookes, and D.-J. Huang, Phys. Rev. Lett. 88, 047201 (2002).

7 J. B. Torrance, M. W. Shafer, and T. R. McGuire, Phys. Rev. Lett. 29, 1168 (1972).

8 B. Laks and C. E. T. G. da Silva, Phys. Rev. Lett. 36, 1204 (1976).

9 A. Mauger, Phys. Rev. B 27, 2308 (1983).

10 P. G. Steeneken, Ph.D. thesis, Rijksuniversiteit Groningen (2002).

11 G. Möller, A. E. Ruckenstein, and S. Schmitt-Rink, Phys.
Rev. B 46, 7427 (1992).

12 G. Pálsson and G. Kotliar, Phys. Rev. Lett. 80, 4775 (1998).

13 A. Mauger, C. Godart, M. Escorne, J. C. Achard, and J. P. Desfours, J. Phys. (Paris) 39, 1125 (1978).

14 G. Czycholl and H. J. Leder, Z. Phys. B 44, 59 (1981).

15 R. Kubo, J. Phys. Soc. Japan 12, 570 (1957).

16 W. Borgiel, T. Herrmann, W. Nolting, and R. Kosimow, Acta Phys. Pol. B 32, 383 (2001).

17 W. Nolting, G. G. Reddy, A. Ramakanth, and D. Meyer, Phys. Rev. B 64, 155109 (2001).

18 W. Nolting and M. Matlak, phys. stat. sol. (b) 123, 155 (1984).

19 V. Velický, S. Kirkpatrick, and H. Ehrenreich, Phys. Rev. 175, 747 (1968).

${ }^{20}$ V. Velický, Phys. Rev. 184, 614 (1969).

21 R. Schiller, Ph.D. thesis, Humboldt-Universität zu Berlin (2000).

22 J. M. D. Coey, M. Viret, and S. von Molnar, Adv. Phys. 48, 167 (1999). 